\title{
MARKED DEPOSITION OF EOSINOPHIL-DERIVED NEUROTOXIN IN ADULT PATIENTS WITH EOSINOPHILIC ESOPHAGITIS
}

\author{
Gail M. Kephart, B.S. ${ }^{1}$, Jeffrey A. Alexander, M.D. ${ }^{2}$, Amindra S. Arora, M.B.B.Chir. ${ }^{2}$, Yvonne \\ Romero, M.D. 2,3,4, Thomas C. Smyrk, M.D. ${ }^{5}$, Nicholas J. Talley, M.D., Ph.D.6, and Hirohito \\ Kita, M.D. 1,3 \\ ${ }_{1}^{1}$ Allergic Diseases Research Laboratory, Mayo Clinic, Rochester, MN, USA \\ 2 Gastroenterology and Hepatology, Mayo Clinic, Rochester, MN, USA \\ ${ }^{3}$ Otolaryngology, Mayo Clinic, Rochester, MN, USA \\ ${ }^{4}$ GI Outcomes Unit, Mayo Clinic, Rochester, MN, USA \\ ${ }^{5}$ Laboratory Medicine and Pathology, Mayo Clinic, Rochester, MN, USA \\ ${ }^{6}$ Gastroenterology and Hepatology, Mayo Clinic, Jacksonville, FL, USA
}

\begin{abstract}
Objective-Eosinophilic esophagitis (EoE) is characterized by infiltration of eosinophils into esophageal epithelium. Blood levels of an eosinophil granule protein, eosinophil-derived neurotoxin (EDN), have been proposed as a biomarker for EoE. However, information regarding localization of EDN in the diseased tissues has not been available. The goal of this study was to evaluate the magnitude and distribution of EDN deposition in tissue specimens from the esophagus of EoE patients.
\end{abstract}

\begin{abstract}
Methods-We studied specimens from 10 adult EoE patients and 8 histologically-normal controls (three under age 17). Sections from mid-esophageal biopsy specimens were stained for EDN by immunofluorescence, using a polyclonal rabbit antibody to EDN. Cellular staining (i.e. infiltration of intact eosinophils) and extracellular staining (i.e. deposition of released EDN) were scored in a blinded manner on an established 7-point scale.
\end{abstract}

\begin{abstract}
Results-Esophageal biopsy specimens from histologically-normal controls showed no or few intact eosinophils and no or minimal extracellular EDN deposition. In contrast, EDN staining was clearly observed in specimens from all EoE patients. In some EoE patients, marked extracellular EDN deposition was observed despite relatively small numbers of intact eosinophils. Overall, there was no correlation between the eosinophil infiltration and the extracellular EDN staining scores.
\end{abstract}

Correspondence/reprint requests: Hirohito Kita, MD, Allergic Diseases Research Laboratory, Mayo Clinic, Rochester, MN 55905, Telephone: (507)284-2511 FAX: (507)284-5045, kita.hirohito@ mayo.edu.

Guarantor of the article: Hirohito Kita, M.D.

Specific author contributions: Acquisition of data, analysis and interpretation of data, and drafting of the manuscript: Gail M. Kephart; conception and design, obtaining funding, patient recruitment, analysis and interpretation of data, manuscript review, and supervision: Jeffrey A. Alexander, M.D.; patient recruitment, manuscript review: Amindra Arora, M.B.B.Chir. and Yvonne Romero, M.D.; pathological interpretation, diagnosis, manuscript review: Thomas C. Smyrk; patient recruitment, analysis and interpretation of data, critical revision of the manuscript: Nicholas J. Talley, M.D.; obtaining funding, acquisition of data, analysis and interpretation of data, critical revision of the manuscript, and supervision: Hirohito Kita, M.D.

Potential competing interests: While several of the authors may be consultants for and/or receiving financial support from private companies, this study is unrelated to any business entity or the doctors' activities other than the general topic of esophageal disease. No potential competing interests exist. 
Conclusions-Marked tissue deposition of extracellular EDN is present in the esophagus of EoE patients. Tissue eosinophil counts may underestimate how extensively eosinophils are involved, particularly in individuals with marked eosinophil degranulation. Evaluation of EDN staining in esophageal biopsy specimens may be useful to diagnose and manage patients with EoE.

\section{Introduction}

Eosinophilic esophagitis (EoE) is a clinicopathologic disease characterized by intense eosinophilic infiltration in the esophageal epithelium, upper gastrointestinal symptoms (dysphagia, food impaction, feeding intolerance, heartburn, etc.), and lack of responsiveness to treatment with high-dose proton pump inhibitors (1-3). While the role of eosinophils in the pathophysiology of EoE is not fully understood, intense eosinophil infiltration of the esophageal epithelium has been used as a diagnostic hallmark of EoE. The minimum number of eosinophils required to diagnose EoE typically ranges from $\geq 15$ to $\geq 30$ in one or more high power fields (HPF) as seen by hematoxylin and eosin (H\&E) staining $(2,4,5)$. Recently, a consensus group proposed a diagnostic guideline of $\geq 15$ eosinophils in one HPF (2). Despite this consensus statement, there seems to be no universal agreement in the medical literature on either the number of eosinophils required to diagnose EoE, the area of the HPF, or the location and number of biopsies used to generate that number $(6,7)$. In addition, EoE is reportedly a patchy disease with variations in both numbers of eosinophils and histologic findings. Among biopsy specimens taken from the same site and even within a given biopsy, the numbers of eosinophils can vary widely(4), creating a challenge for the accurate diagnosis of EoE.

Eosinophil granules contain several cytotoxic proteins, including eosinophil-derived neurotoxin (EDN). Cellular activation with appropriate stimuli leads to extracellular release of the granule proteins (8), and extracellular EDN has been used as an indicator of eosinophil activation and degranulation in vitro (9). Clinically, increased levels of EDN in body fluids have been observed in patients with various eosinophil-associated diseases. For example, in children with asthma, EDN levels are elevated in serum and urine (10). In patients with inflammatory bowel disease, EDN levels in whole gut lavage fluids may be a more stable marker of eosinophil degranulation than eosinophil major basic protein (MBP) levels (11). A recent EoE study showed that plasma EDN levels correlate with esophageal eosinophil density (12). Furthermore, plasma EDN levels have been proposed as a noninvasive biomarker for diagnosing or monitoring EoE $(2,12)$. Surprisingly, there were no differences in fecal EDN levels in EoE patients compared to unaffected controls (12), raising fundamental questions as to whether EDN is present or released in the digestive tracts of patients with active EoE. No previous reports have examined the presence of EDN in esophageal tissues from EoE patients or characterized the magnitude and distribution of EDN deposition among them.

Therefore, we examined esophageal biopsy specimens from EoE patients and histologicallynormal controls for EDN by immunofluorescence. In the majority of EoE patients, we found marked deposition of extracellular EDN; in histologically-normal controls, EDN staining was minimal or absent. Importantly, specimens from some EoE patients showed marked extracellular EDN deposition despite relatively small numbers of intact eosinophils. Thus, in certain EoE patients, the number of eosinophils in esophageal specimens may underestimate the magnitude of eosinophil involvement in their esophageal pathology.

\section{Methods}

\section{Study Population}

Four mid-esophageal biopsies (10-15 $\mathrm{cm}$ above the gastroesophageal border) were obtained from 10 patients with EoE and embedded in one paraffin block. Their mean age was 41 (29- 
49) years, and nine were male. Inclusion criteria were: $>17$ years of age, $\geq 20$ eosinophils/HPF (vide infra), and dysphagia. Each patient completed a validated dysphagia questionnaire (13). This study was performed before the consensus definition (2) of EoE requiring a normal $\mathrm{pH}$ monitoring study of the distal esophagus and failure of symptoms to respond to PPI therapy was established. Therefore, we used the criterion of $\geq 20$ eosinophils/HPF (maximum density) to minimize the possibility of including patients with gastroesophageal reflux disease (GERD). Exclusion criteria were: systemic or topical steroid use in the past three months, previous dietary or pharmacologic therapy for EoE, erosive esophagitis, and dilatation of esophagus at the time of index endoscopy.

One section from the paraffin block of each patient was stained with H\&E and read by a GI pathologist (TS). The area of greatest eosinophil density within the four biopsies was located by low-power review. Then, within this area, eosinophils were counted in the one HPF containing the greatest density of eosinophils, using a Nikon E600 microscope with a 40X objective field diameter of $0.6 \mathrm{~mm}$ and a field area of $0.31 \mathrm{~mm}^{2}$ (Table 1). The mean number of eosinophils/HPF (maximum density) for the 10 EoE patients was 53 (range 25-100). Five patients had a PPI trial that did not resolve their dysphagia symptoms. All patients had solid food dysphagia that subsequently responded to topical steroid therapy. Three of the patients had heartburn. Endoscopic findings of EoE (rings, furrows, or white spots) were seen in seven patients. None of the patients had esophageal strictures (endoscopically-seen narrowing of > $4 \mathrm{~mm})$.

In addition, mid-esophageal biopsy specimens were obtained from eight histologically-normal controls; their mean age was 39 (10-70) years, three were under age 17, and four were male. Four controls had dysphagia, and five controls had heartburn or regurgitation symptoms. These biopsy specimens were histologically normal, i.e. no pathological abnormalities were present on H\&E stained sections, and no eosinophils were present. All controls had a normal endoscopy.

\section{Immunofluorescence Staining of Tissue Specimens}

Serial sections from the mid-esophageal specimen of each patient and control were examined by immunofluorescence to detect EDN or major basic protein (MBP) as described previously (14-18). Briefly, sections were deparaffinized, rehydrated, incubated with $0.1 \%$ trypsin (Sigma, St. Louis, MO) to expose antigenic sites, and blocked overnight in $10 \%$ normal goat serum (Pel-Freez Biologicals, Rogers, AR). After washing the next day, the slides were incubated with either polyclonal rabbit anti-human EDN, or affinity-purified polyclonal rabbit anti-human MBP (both prepared at Mayo Clinic Rochester), or normal rabbit serum (NRS) (Pel Freez) as a control. The slides were then washed and incubated in $1 \%$ chromotrope $2 \mathrm{R}$ (Sigma) to block nonspecific staining of eosinophils by the fluorescein-labeled secondary antibody. The slides were washed again and incubated with fluorescein-labeled goat anti-rabbit IgG (Jackson ImmunoResearch, West Grove, PA). After a final wash, the slides were mounted with Vectashield mounting medium (Vector Laboratories, Inc., Burlingame, CA), coverslipped, and sealed.

\section{Specificity of EDN antibody}

The specificity of the EDN antibody was examined by a series of immunoabsorption experiments. EDN protein and human serum albumin (HSA, Sigma) were coupled to cyanogen bromide-activated Sepharose 4B (Sigma) as previously described (19). Briefly, $2 \mathrm{mg}$ of each protein were added to $2 \mathrm{~mL}$ activated Sepharose and rotated for 24 hours at $4^{\circ} \mathrm{C}$. An additional $2 \mathrm{~mL}$ aliquot of activated Sepharose with no protein was rotated similarly. The remaining active sites were blocked with lysine monohydrochloride, and ionically-bound protein was removed by alternating washes of acetate buffer $(\mathrm{pH}=4)$ and coupling buffer $(\mathrm{pH}=8)$. Two $\mathrm{mL}$ of a 
1:10 dilution of the EDN antibody was added to each protein-coupled Sepharose and uncoupled Sepharose and rotated as above. The resulting supernatants were tested by immunofluorescence on sections from two EoE patient biopsy specimens at a 1:80 dilution, the same dilution used for the unabsorbed EDN antibody.

\section{Analysis of specimens}

The immunofluorescent-stained slides were examined at X160 (Zeiss 16X objective, field diameter $=1.5 \mathrm{~mm}$ ) with an Axiophot fluorescence microscope (Carl Zeiss, Inc., Oberkochen, Germany), equipped with an AxioCam HRc digital camera and AxioVision 4.2 software. By using a 16X objective, we examined an area nearly six-fold larger $\left(1.76 \mathrm{~mm}^{2}\right)$ than that encompassed with a Nikon X 40 objective $\left(0.31 \mathrm{~mm}^{2}\right)$. We evaluated the two largest biopsies in the paraffin block from each patient and control because these biopsies would have the least amount of crush artifact, if any. To evaluate the amount of EDN staining in the specimens objectively and reproducibly, a scoring system similar to that used for MBP staining in stomach and small bowel endoscopic biopsy specimens (20) was developed. Representative photomicrographs (X160) were taken to document the spectra of eosinophil infiltration and extracellular EDN deposition in esophageal biopsy specimens. Photomicrographs that illustrated degrees of either primarily eosinophil infiltration or primarily extracellular EDN deposition were selected and assigned scores of 0 (none), 1 (minimal), 2 (moderate), and 3 (confluent); these photographs became the standards used in the scoring procedure (Figure 5, vide infra). Using these scoring standards and a 16X objective, we evaluated cellular EDN staining (i.e. eosinophil infiltration) in the biopsies on a 7-point scale from 0 to 3 in increments of 0.5; extracellular EDN staining (i.e. deposition of released EDN) was scored similarly. To obtain mean scores for eosinophil infiltration and extracellular EDN deposition, the two largest biopsies from each paraffin block were scored independently by two investigators (GMK and HK) who were unaware of the diagnosis. The investigators' scores for the two biopsies from each patient were averaged, and an overall mean value was calculated (see footnote in Table 1 for an example of the calculations).

\section{Statistical analysis}

Correlation between the eosinophil infiltration scores and extracellular EDN deposition scores was analyzed by Spearman rank, using a statistical software package (Instat 3.0, GraphPad Software Abacus Concepts, La Jolla, CA).

\section{Results}

\section{Deposition of EDN is more pronounced than MBP in EoE patient biopsy specimens}

In studies of asthma and atopic dermatitis, MBP has been used as a marker of eosinophil infiltration and degranulation $(15,21)$. Therefore, we compared MBP and EDN staining in serial sections of an esophageal biopsy specimen from an EoE patient. While the patterns of the cellular EDN (Figure 1A) and MBP (Figure 1B) staining were roughly similar, the patterns of the extracellular staining were strikingly different. The extracellular MBP staining was largely granular, while the extracellular EDN deposition was diffuse and covered a larger area compared to the extracellular MBP staining. Serial sections were stained with H\&E (Figure 1C) and NRS (Figure 1D); the NRS-stained section was negative. A specimen from another EoE patient showed similar findings.

To verify the specificity of EDN staining, we performed a series of immunoabsorption studies. Absorption of EDN antibody with either uncoupled Sepharose or HSA-Sepharose did not affect the immunoreactivity, as EoE patient biopsy sections stained with these absorbed antibodies showed staining similar to that of the unabsorbed EDN antibody. In contrast, absorption of EDN antibody with EDN-Sepharose completely removed the immunoreactivity; weak 
fluorescence from what appears to be autofluorescence of red blood cells was the only signal detectable (see Supplemental Figure 1 and Supplemental Figure 2 online). These results, along with data demonstrating the purity of the EDN used to immunize the rabbits, show that the anti-EDN antibody is specific for EDN (22). Therefore, we examined the use of EDN as a marker of eosinophil involvement in esophageal biopsy specimens from $10 \mathrm{EoE}$ patients and 8 histologically-normal controls.

\section{EoE patients' biopsy specimens show marked EDN deposition with three distinct patterns}

Esophageal biopsy specimens from histologically-normal controls showed very few or no intact eosinophils; likewise, extracellular EDN deposition was minimal (Figure 2A-F). In contrast, biopsy specimens from EoE patients showed marked cellular and extracellular EDN staining (Figure 3). Three different patterns of EDN staining were observed in EoE patients: primarily cell-associated, a combination of cell-associated and extracellular EDN deposition, and primarily extracellular EDN deposition. For example, specimens from 2/10 patients demonstrated primarily eosinophil infiltration with minimal extracellular EDN deposition (Figure 3A, 3B). Figure 3B is an H\&E counterstain of Figure 3A and illustrates that the cells staining with the EDN antibody are eosinophils (white and black arrows). Biopsy specimens from 6/10 patients showed both eosinophil infiltration and extracellular EDN deposition (Figure 3C, 3D). Interestingly, biopsy specimens from $2 / 10$ patients showed widespread diffuse extracellular EDN deposition with minimal eosinophil infiltration (Figure 3E-3H). Diffuse extracellular EDN deposition was often seen throughout the esophageal epithelium (Figure 3C), while in some patients, intense extracellular EDN deposition was observed on the luminal surface and in the superficial mucosa (Figure 3E). Figure 3G (higher magnification of Figure 3E) clearly shows extensive extracellular EDN deposition, particularly along the luminal surface of epithelial cells (white arrowhead), in spite of relatively few intact eosinophils on the serial H\&E stain (black arrowhead, Figure $3 \mathrm{H}$ ). Serial sections to those shown in Figure $3 \mathrm{C}$ and $3 \mathrm{E}$ were stained with NRS plus FITC-conjugated goat anti-rabbit IgG and showed no visible fluorescence signal (see Supplemental Figure 3 online). Furthermore, consistent with the patchy nature of EoE histology, heterogeneous EDN staining was seen not only among different biopsies taken from the same site, but also within the same biopsy. For example, within a given biopsy, a few eosinophils and minimal extracellular deposition could be present in one X160 field (Figure 4A), while extensive extracellular EDN deposition could be present in an adjacent X160 field (Figure 4B).

\section{Scoring of intracellular and extracellular EDN staining}

Thus, all EoE biopsy specimens showed EDN staining; however, the pattern and the distribution of the EDN staining among the EoE patients were heterogeneous. Therefore, to evaluate the deposition of EDN systematically and objectively, we developed a scoring system (Figure 5) to analyze semiquantitatively the amount of EDN staining. The scoring system was based on standards (X160 photomicrographs) that represented the various magnitudes (Grade 1, minimal; Grade 2, moderate; and Grade 3, confluent) of both cellular and extracellular EDN deposition. By comparison to these standards, two observers examined the two largest biopsies of each mid-esophageal specimen and assigned scores for both eosinophil infiltration (intracellular EDN) and extracellular EDN deposition. The scores of the two observers were averaged to obtain a mean score for each biopsy (e.g. Biopsy \#1 and Biopsy \#2), and the scores from the two biopsies were averaged to obtain overall scores for eosinophil infiltration and extracellular EDN deposition for each mid-esophageal specimen.

In the biopsy specimens from histologically-normal controls, scores for both eosinophil infiltration and extracellular EDN deposition were $\leq 0.5$ in all specimens (Table 1). In contrast, in the EoE patients, marked eosinophil infiltration and/or abundant extracellular EDN deposition were evident with mean scores of 1.8 and 2.1, respectively (Table 1). Biopsy 
specimens from $6 / 10$ EoE patients had mean scores of $\geq 1.5$ for both cellular and extracellular EDN staining. In contrast, biopsy specimens from 2/10 EoE patients (patients 1 and 8) showed primarily intact eosinophil infiltration (mean scores of 2.3 and 1.8, respectively) and minimal extracellular EDN deposition (mean scores of 1.0). Biopsy specimens from another 2/10 EoE patients (patients 4 and 10) showed extracellular EDN deposition (mean scores of 2.3 and 2.5, respectively) and only minimal eosinophil infiltration (mean scores of 1.2 and 1.3, respectively). Figure 6 shows a comparison between eosinophil infiltration scores and extracellular EDN deposition scores of all EoE patient biopsy specimens. Overall, there was no correlation between eosinophil infiltration and extracellular EDN deposition $(\mathrm{R}=-0.0067$, $\mathrm{p}=0.9790)$.

\section{Discussion}

Development of sensitive and reliable biomarkers for EoE is critical for the diagnosis and management of EoE patients and for patients enrolled in clinical trials of EoE. Plasma EDN levels correlate with esophageal eosinophil counts and have been proposed as a noninvasive biomarker for diagnosing or monitoring $\operatorname{EoE}(2,12)$. However, there were no differences in fecal EDN levels in EoE patients compared to normal controls (12). In this study, we stained esophageal biopsy specimens from EoE patients for EDN by immunofluorescence to evaluate the magnitude and distribution of both eosinophil infiltration and extracellular EDN deposition.

No or minimal EDN staining (mean scores of $\leq 0.5$ for both cellular and extracellular deposition) was found in esophageal biopsy specimens from histologically-normal controls, consistent with previous observations that eosinophils are not present in the normal esophagus $(23,24)$. In contrast, eosinophil infiltration and extracellular EDN deposition were regularly seen in EoE patients. Importantly, the extent of extracellular EDN deposition did not correlate with the numbers of infiltrating eosinophils (Figures 3 and 6). For example, marked eosinophil infiltration was observed with minimal extracellular EDN deposition (Figure 3A, 3B), while in others, marked extracellular EDN deposition was observed with relatively small numbers of intact eosinophils (Figure 3E, 3F). Furthermore, both eosinophil infiltration and extracellular EDN deposition were heterogeneous even within a given specimen (Figure 4). Previously, a study of skin biopsy specimens from patients with atopic dermatitis showed deposition of eosinophil granule proteins in the dermis in the virtual absence of intact eosinophils (21). Thus, using a given number of intact eosinophils in an H\&E-stained section as a diagnostic cut-off could underestimate the extent of eosinophil inflammation. More comprehensive approaches to analyze both the numbers of infiltrating eosinophils as well as the magnitude of deposition of eosinophil-derived products (e.g. EDN) may be necessary to diagnose EoE accurately.

The reason(s) for more marked eosinophil degranulation in the esophagus of some EoE patients but not in others is unknown. Receptors for cytokines, chemokines, complement, immunoglobulins, and proteases allow eosinophils to interact with the extracellular milieu (25). In particular, Th2 cytokines, such as IL-5 and IL-13, and eotaxin-3 are implicated in the pathophysiology of EoE (3); in vitro exposure of eosinophils to these cytokines/chemokines induces degranulation (8). Furthermore, systemic expression of IL-5 and epithelial expression of eotaxin-2 induced extensive eosinophil degranulation as well as structural damage and remodeling in mouse airways in vivo (26). In addition, when EoE patients were treated with anti-IL-5 antibody (mepolizumab), both blood eosinophil numbers and cell activation decreased (27). Thus, eosinophil degranulation and the subsequent deposition of EDN in the esophagus may reflect tissue levels of IL-5 and eotaxin and may represent a useful marker for the immunological disease activity of EoE.

Once released extracellularly, EDN could activate human dendritic cells (DCs), with subsequent production of inflammatory mediators (28). In mice, EDN can stimulate DC 
maturation through the TLR2 signaling pathway and enhance antigen-specific Th2 immune responses, suggesting that EDN may act as an alarmin $(29,30)$. Furthermore, EDN can be toxic to certain mammalian cells (31). While MBP and EDN are both presumably products of eosinophil degranulation, we also found distinctive differences between EDN and MBP staining (Figure 1). Although the reasons for this observation are unknown, the biochemical differences between MBP and EDN might provide an explanation. MBP is a highly basic and amphipathic protein; it readily precipitates at neutral $\mathrm{pH}$ (32). In contrast, the isoelectric point of EDN is closer to neutral, and it does not readily precipitate from neutral solutions. Therefore, once released from eosinophils, EDN may diffuse further into the tissues than MBP. Another possibility is the so-called "differential degranulation of eosinophils" mechanism; under certain experimental conditions, eosinophils appear to release one granule protein but not another (33). A similar mechanism may occur in vivo in patients with EoE. Further studies are needed to elucidate the mechanisms for EDN release by eosinophils in esophageal mucosa and to investigate the role of released EDN in the pathophysiology of EoE.

We recognize several limitations in this study. The sample size is small. The EoE patients and histologically-normal controls are not matched for sex, age, or medications. However, our observations of minimal or no EDN staining in esophageal specimens from histologicallynormal controls are consistent with previous findings that indicate few or no eosinophils are present in normal esophageal mucosa $(23,24)$. Furthermore, we studied specimens from only one esophageal site (mid-esophagus) from each patient and control. Using the criterion of $\geq 15$ eosinophils/HPF, Gonsalves et al showed that five biopsies are needed to obtain a diagnostic sensitivity of $100 \%$ for EoE (34). Given the heterogeneous nature of the eosinophil distribution in EoE, a recent review article recommended obtaining four biopsy specimens from both the proximal and the distal esophagus to maximize diagnostic sensitivity (35). Finally, as mentioned in the Methods section, this study was performed before the consensus definition of EoE was established. Therefore, our study may have included patients with GERD.

However, all patients had topical steroid-responsive dysphagia, most had typical endoscopic findings of EoE, and half (including all those with heartburn or regurgitation symptoms) had a PPI trial without relief of their dysphagia.

In conclusion, we found that localization of EDN may be a useful biomarker of eosinophilic inflammation in esophageal tissues from EoE patients. Rather than counting eosinophils by $\mathrm{H} \& \mathrm{E}$ staining, could evaluation of EDN staining (which detects both eosinophil infiltration and degranulation and assesses a larger area of an esophageal biopsy specimen) reduce the number of biopsy specimens necessary to make an accurate diagnosis of EoE? Alternatively, would EDN deposition in esophageal tissues better reflect the extent of immunological disease in EoE than eosinophil numbers determined by H\&E staining? Further studies of EDN staining in patients with both minimal eosinophilia on H\&E staining and clinical symptoms of EoE and in those patients with GERD are needed to evaluate the potential utility of EDN staining to diagnose patients whose disease status is presently ambiguous.

\section{Study Highlights}

\section{WHAT IS CURRENT KNOWLEDGE}

- Eosinophilic esophagitis (EoE) shows intense eosinophilic infiltration in the epithelium.

- The sensitivity of $\geq 15$ eosinophils/HPF from esophageal mucosa for this clinical syndrome is unknown.

- Eosinophil-derived neurotoxin (EDN) is increased in body fluids from patients with eosinophil-associated diseases. 


\section{WHAT IS NEW HERE}

- Biopsy specimens from EoE patients showed both extracellular and intact eosinophil staining for EDN, but histologically-normal controls did not.

- Extracellular EDN deposition was seen in some EoE patient biopsies that contained few intact eosinophils.

- Reliance on eosinophil counts may overlook EoE patient biopsies with marked eosinophil degranulation but few intact eosinophils.

- Localization of EDN in esophageal biopsy specimens may be useful to evaluate eosinophilic inflammation in EoE.

\section{Supplementary Material}

Refer to Web version on PubMed Central for supplementary material.

\section{Acknowledgments}

The authors thank Cheryl R. Adolphson for editorial assistance and LuRaye S. Eischens for secretarial assistance.

Financial support: National Institutes of Health Grant AI 34486 (HK) and the Mayo Foundation.

Supported in part by National Institutes of Health Grant AI 34486 and the Mayo Foundation

\section{References}

1. Attwood SEA, Smyrk TC, Demeester TR, et al. Esophageal eosinophilia with dysphagia: A distinct clinicopathologic syndrome. Dig Dis Sci 1993;38:109-16. [PubMed: 8420741]

2. Furuta GT, Liacouras CA, Collins MH, et al. Eosinophilic esophagitis in children and adults: A systematic review and consensus recommendations for diagnosis and treatment. Gastroenterology 2007;133:1342-63. [PubMed: 17919504]

3. Blanchard C, Wang N, Rothenberg ME. Eosinophilic esophagitis: Pathogenesis, genetics, and therapy. J Allergy Clin Immunol 2006;118:1054-59. [PubMed: 17088129]

4. Collins MH. Histopathologic features of eosinophilic esophagitis. Gastrointest Endoscopy Clin N Am 2008;18:59-71.

5. Chang F, Anderson S. Clinical and pathological features of eosinophilic oesophagitis: a review. Pathology 2008;40:3-8. [PubMed: 18038308]

6. Dellon ES, Aderoju A, Woosley JT, et al. Variability in diagnostic criteria for eosinophilic esophagitis: A systematic review. Am J Gastroenterol 2007;102:2300-13. [PubMed: 17617209]

7. Ravi K, Alexander JA, Neumann DA, et al. Low grade esophageal eosinophilia in adults: An unrecognized part of the spectrum of eosinophilic esophagitis? Am J Gastroenterol 2007;102(Suppl 2):S129.

8. Kita, H.; Adolphson, CR.; Gleich, GJ. Biology of eosinophils. In: Adkinson, NF., Jr; Yunginger, JW.; Busse, WW.; Bochner, BS.; Holgate, ST.; Simons, FER., editors. Middleton's Allergy Principles and Practice. 6. Philadelphia: Mosby; 2003. p. 305-32.

9. Abu-Ghazaleh RI, Fujisawa T, Mestecky J, et al. IgA-induced eosinophil degranulation. J Immunol 1989;142:2393-2400. [PubMed: 2926137]

10. Koller DY, Halmerbauer G, Frischer T, et al. Assessment of eosinophil granule proteins in various body fluids: Is there a relation to clinical variables in childhood asthma? Clin Exp Allergy 1999;29:786-93. [PubMed: 10336595]

11. Levy AM, Gleich GJ, Sandborn WJ, et al. Increased eosinophil granule proteins in gut lavage fluid from patients with inflammatory bowel disease. Mayo Clin Proc 1997;72:117-23. [PubMed: 9033543] 
12. Konikoff MR, Blanchard C, Kirby C, et al. Potential of blood eosinophils, eosinophil-derived neurotoxin, and eotaxin-3 as biomarkers of eosinophil esophagitis. Clin Gastroenterol Hepatol 2006;4:1328-36. [PubMed: 17059896]

13. Grudell ABM, Alexander JA, Enders FB, et al. Validation of the Mayo dysphagia questionnaire. Diseases of the Esophagus 2007;20:202-5. [PubMed: 17509115]

14. Perez GL, Peters MS, Reda AM, et al. Mast cells, neutrophils, and eosinophils in prurigo nodularis. Arch Dermatol 1993;129:861-5. [PubMed: 8323307]

15. Filley WV, Holley KE, Kephart GM, et al. Identification by immunofluorescence of eosinophil granule major basic protein in lung tissues of patients with bronchial asthma. Lancet 1982;2:11-6. [PubMed: 6177986]

16. Peters MS, Rodriguez M, Gleich GJ. Localization of human eosinophil granule major basic protein, eosinophil cationic protein, and eosinophil-derived neurotoxin by immunoelectron microscopy. Lab Invest 1986;54:656-62. [PubMed: 3520144]

17. Ott NL, Gleich GJ, Peterson EA, et al. Assessment of eosinophil and neutrophil participation in atopic dermatitis: comparison with the IgE-mediated late-phase reaction. J Allergy Clin Immunol 1994;94:120-8. [PubMed: 8027490]

18. Borrego L, Maynard B, Peterson EA, et al. Deposition of eosinophil granule proteins precedes blister formation in bullous pemphigoid. Comparison with neutrophil and mast cell granule proteins. Am J Pathol 1996;148:897-909. [PubMed: 8774144]

19. Filley WV, Ackerman SJ, Gleich GJ. An immunofluorescent method for specific staining of eosinophil granule major basic protein. J Immunol Meth 1981;47:227-238.

20. Talley NJ, Kephart GM, McGovern TW, et al. Deposition of eosinophil granule major basic protein in eosinophilic gastroenteritis and celiac disease. Gastroenterology 1992;103:137-45. [PubMed: 1612323]

21. Leiferman KM, Ackerman SJ, Sampson HA, et al. Dermal deposition of eosinophil-granule major basic protein in atopic dermatitis. Comparison with onchocerciasis. N Engl J Med 1985;313:282-5. [PubMed: 3892296]

22. Gleich GJ, Loegering DA, Bell MP, et al. Biochemical and functional similarities between human eosinophil-derived neurotoxin and eosinophil cationic protein: homology with ribonuclease. Proc Natl Acad Sci U S A 1986;83:3146-50. [PubMed: 3458170]

23. Kato M, Kephart GM, Talley NJ, et al. Eosinophil infiltration and degranulation in normal human tissue. Anatomical Record 1998;252:418-25. [PubMed: 9811220]

24. Rothenberg ME, Mishra A, Collins MH, et al. Pathogenesis and clinical features of eosinophilic esophagitis. J Allergy Clin Immunol 2001;108:891-4. [PubMed: 11742263]

25. Hogan SP, Rosenberg HF, Moqbel R, et al. Eosinophils: biological properties and role in health and disease. Clin Exp Allergy 2008;38:709-50. [PubMed: 18384431]

26. Ochkur SI, Jacobsen EA, Protheroe CA, et al. Co-expression of IL-5 and eotaxin-2 in mice creates an eosinophil-dependent model of respiratory inflammation with characteristics of severe asthma. $\mathrm{J}$ Immunol 2007;178:7879-89. [PubMed: 17548626]

27. Stein ML, Villanueva JM, Buckmeier BK, et al. Anti-IL-5 (mepolizumab) therapy reduces eosinophil activation ex vivo and increases IL-5 and IL-5 receptor levels. J Allergy Clin Immunol 2008;121:1473-83. [PubMed: 18410960]

28. Yang D, Chen Q, Rosenberg HF, et al. Human ribonuclease A superfamily members, eosinophilderived neurotoxin and pancreatic ribonuclease, induce dendritic cell maturation and activation. $\mathrm{J}$ Immunol 2004;173:6134-42. [PubMed: 15528350]

29. Yang D, Chen Q, Su SB, et al. Eosinophil-derived neurotoxin acts as an alarmin to activate the TLR2MyD88 signal pathway in dendritic cells and enhances Th2 immune responses. J Exp Med 2008;205:79-90. [PubMed: 18195069]

30. Bird L. Allergy and asthma: calling all $\mathrm{T}_{\mathrm{H}} 2$ cells. Nature Reviews Immunol 2008;8:162.

31. Newton DL, Walbridge S, Mikulski SM, et al. Toxicity of an antitumor ribonuclease to Purkinje neurons. J Neurosci 1994;14:538-44. [PubMed: 8301353]

32. Gleich GJ. Mechanisms of eosinophil-associated inflammation. J Allergy Clin Immunol 2000;105:651-63. [PubMed: 10756213] 
33. Gounni AS, Lamkhioued B, Ochiai K, et al. High-affinity IgE receptor on eosinophils is involved in defence against parasites. Nature 1994;367:183-6. [PubMed: 8114916]

34. Gonsalves N, Policarpio-Nicolas M, Zhang Q, et al. Histopathologic variability and endoscopic correlates in adults with eosinophilic esophagitis. Gastrointest Endosc 2006;64:313-9. [PubMed: 16923475]

35. Gonsalves N. Eosinophilic esophagitis: History, nomenclature, and diagnostic guidelines. Gastrointest Endoscopy Clin N Am 2008;18:1-9. 

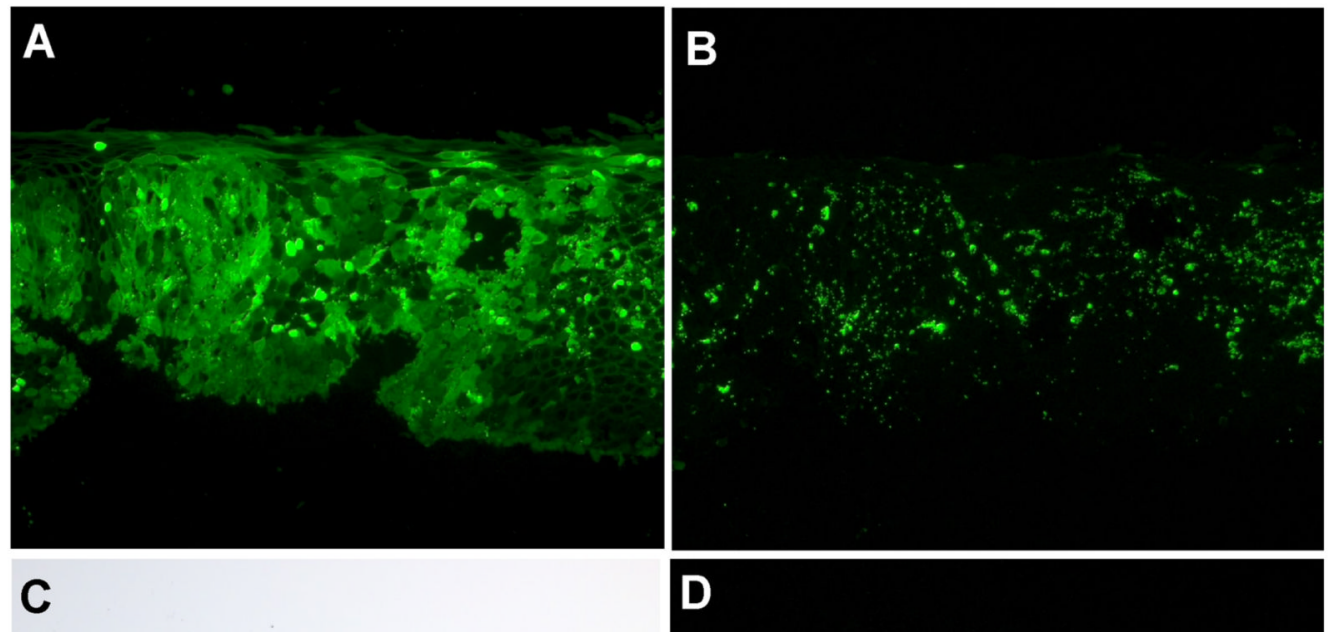

D

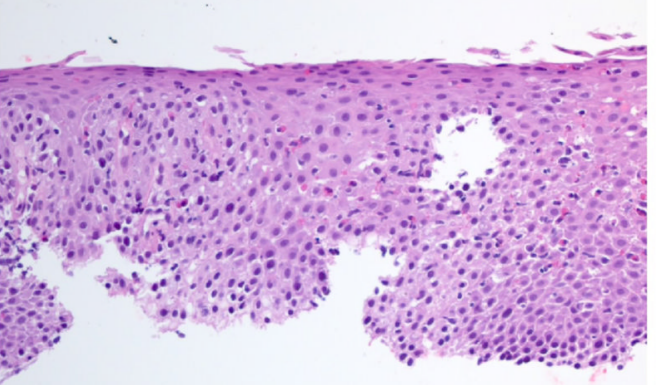

\section{D}

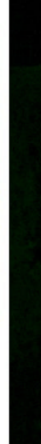

\section{Figure 1.}

Comparison of EDN and MBP staining in an EoE patient esophageal biopsy specimen. Serial sections of a specimen from Patient 6 (Table 1) were stained as follows: A, rabbit anti-human EDN; B, affinity-purified rabbit anti-human MBP; C, H\&E; and D, NRS as a negative control. Original magnification, X160. 

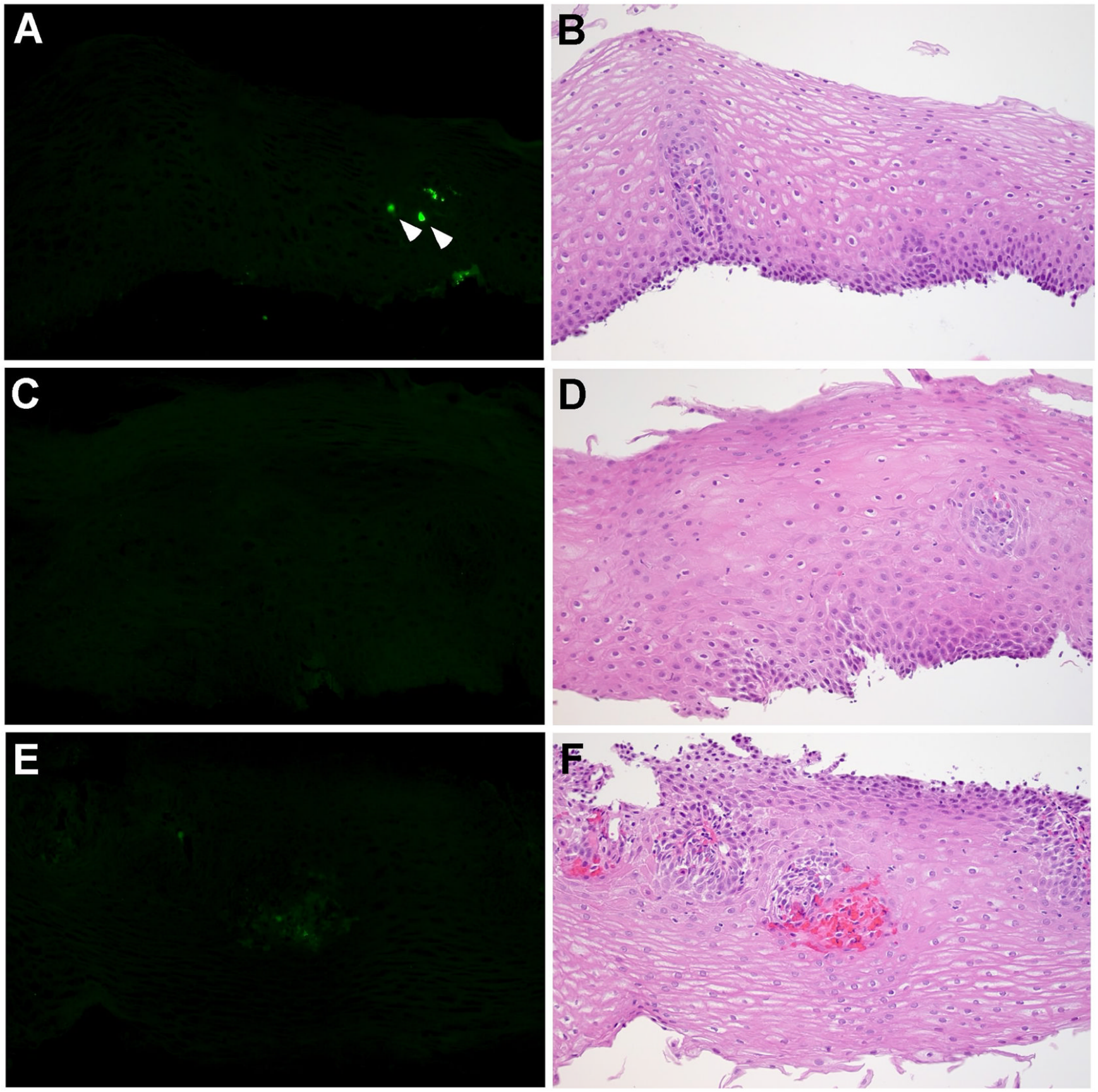

Figure 2.

Localization of EDN in histologically-normal control esophageal biopsy specimens. The sections from 3 histologically-normal controls are stained with anti-EDN. A, C, and E, rabbit anti-human EDN; B, D, and F, H\&E. The arrowheads in A identify intact eosinophils. Original magnification, X160. 

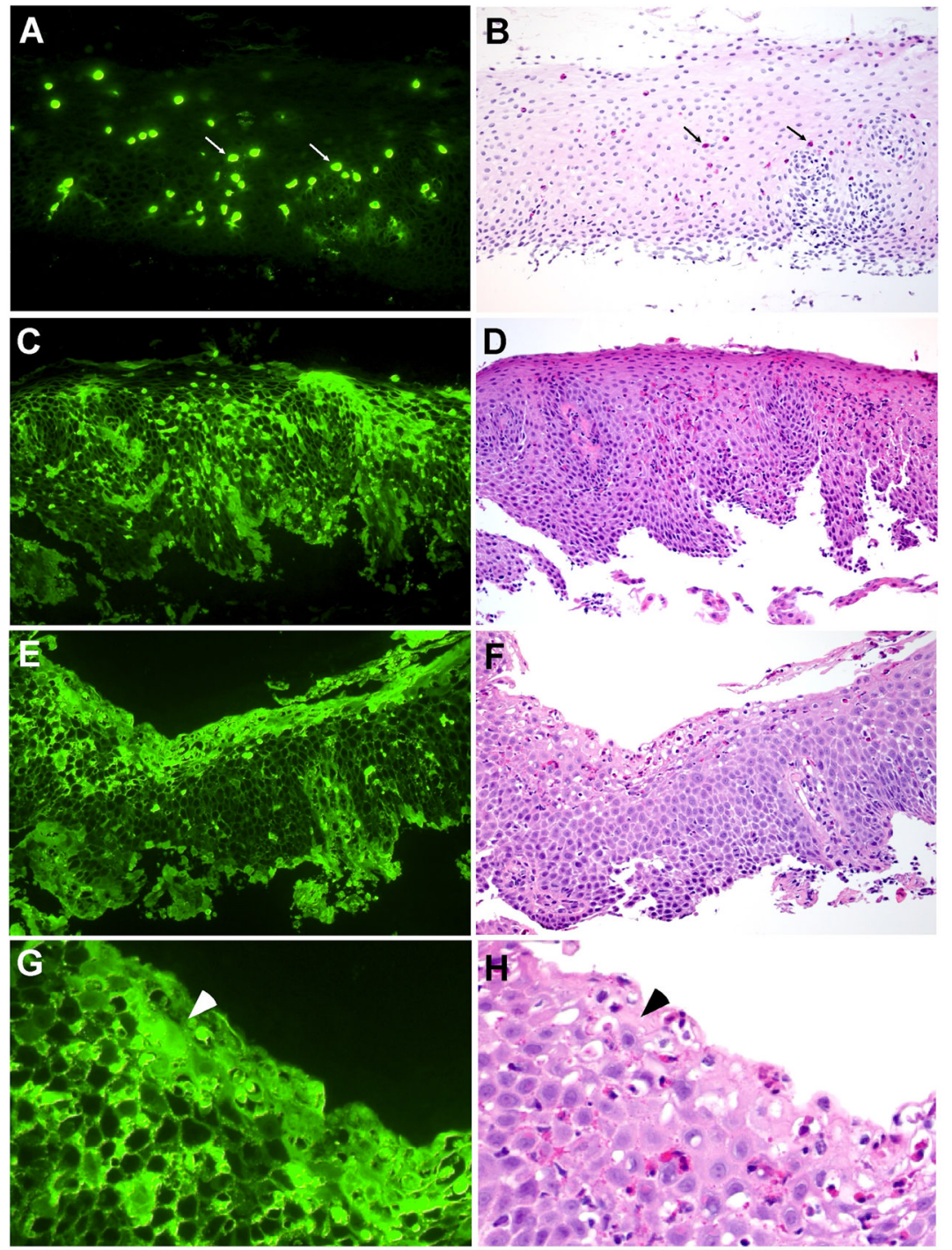

Figure 3.

Localization of EDN in EoE patient esophageal biopsy specimens. A, C, E, and G, sections stained with anti-EDN. B, D, F, and $\mathbf{H}$, sections stained with H\&E. Panel $\mathbf{B}$ is an H\&E counterstain of the same section shown in Panel A.; therefore, the black arrows in $\mathbf{B}$ identify the same eosinophils shown by the white arrows in $\mathbf{A . D}, \mathbf{F}$, and $\mathbf{H}$, serial sections to $\mathbf{C ,} \mathbf{E}$, and $\mathbf{G}$, respectively. $\mathbf{G}$ and $\mathbf{H}$ are a higher magnification of $\mathbf{E}$ and $\mathbf{F}$, respectively; the arrowheads identify an area of intense extracellular EDN deposition. Original magnification, $\mathrm{X} 160$. 


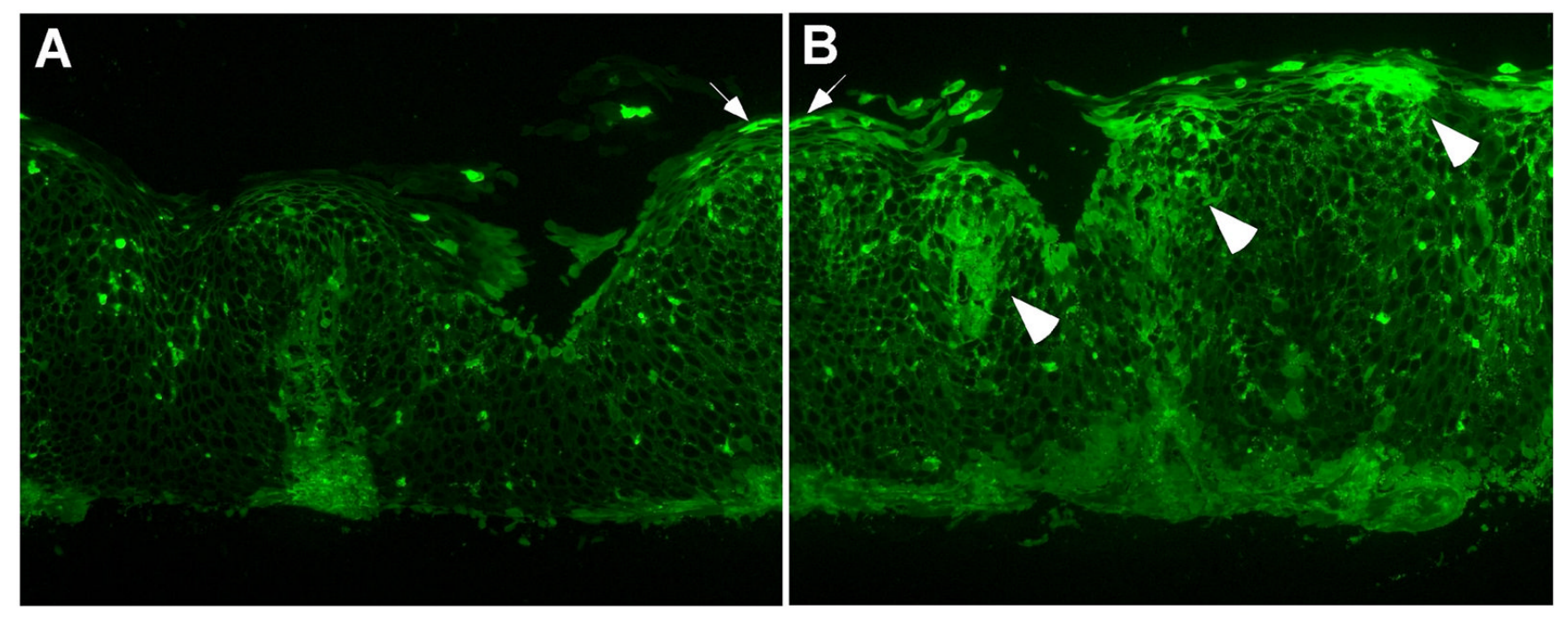

Figure 4.

Heterogeneity of EDN staining in an EoE patient esophageal biopsy specimen. A and $\mathbf{B}$, section from Patient 5 (Table 1) stained with anti-EDN. B is one X160 field to the right of $\mathbf{A}$; the arrows in $\mathbf{A}$ and $\mathbf{B}$ point to the same cell. The arrowheads in $\mathbf{B}$ identify areas of EDN deposition. Original magnification, X160. 

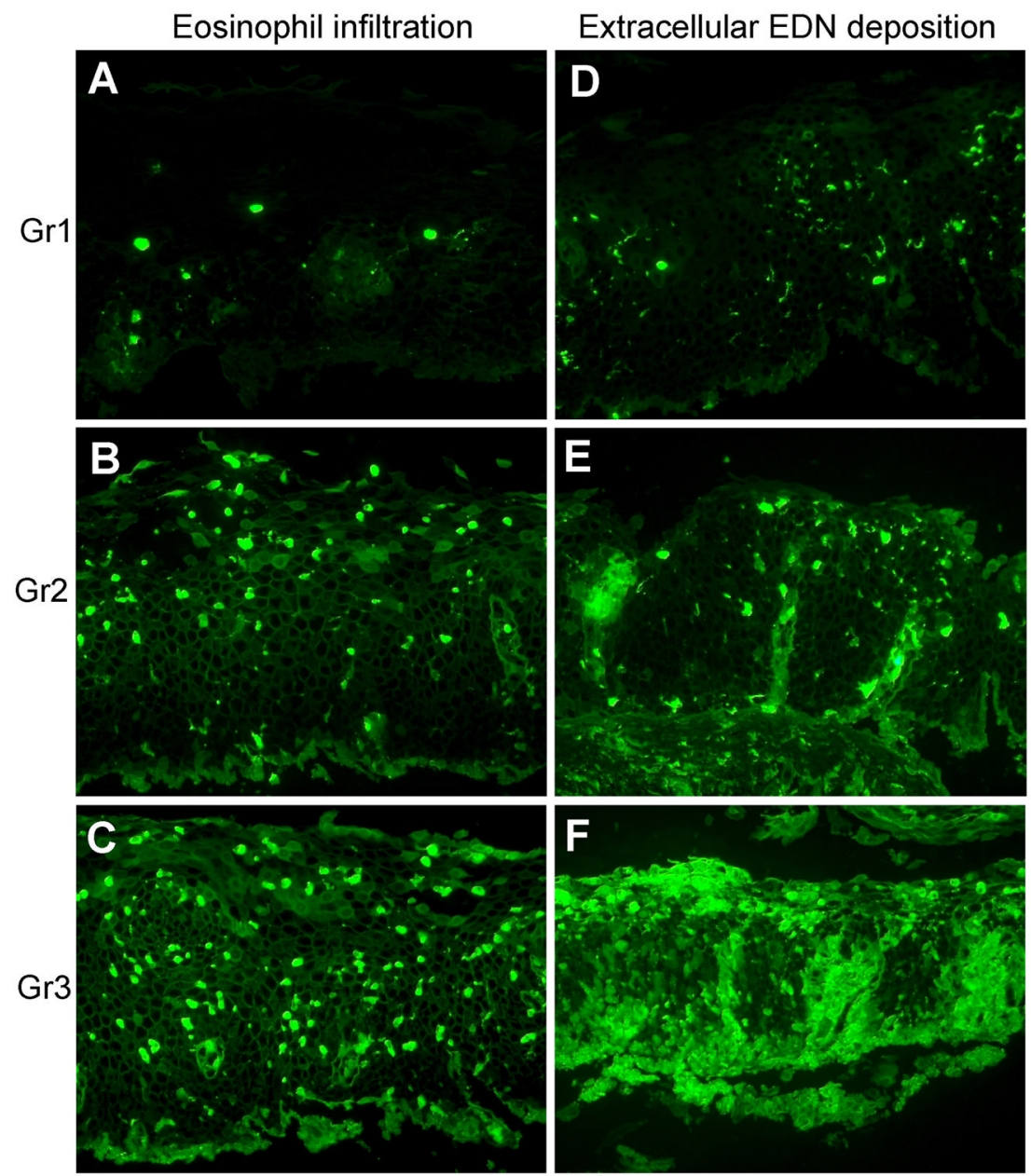

Figure 5.

Scoring of eosinophil infiltration and extracellular EDN deposition in esophageal biopsy specimens. A-F, sections stained with anti-EDN. A, B, and $\mathbf{C}$ show representative examples of eosinophil infiltration scored as 1, 2, or 3, respectively. D, E, and $\mathbf{F}$ show representative examples of extracellular EDN deposition scored as 1, 2, or 3, respectively. Original magnification, X160. 


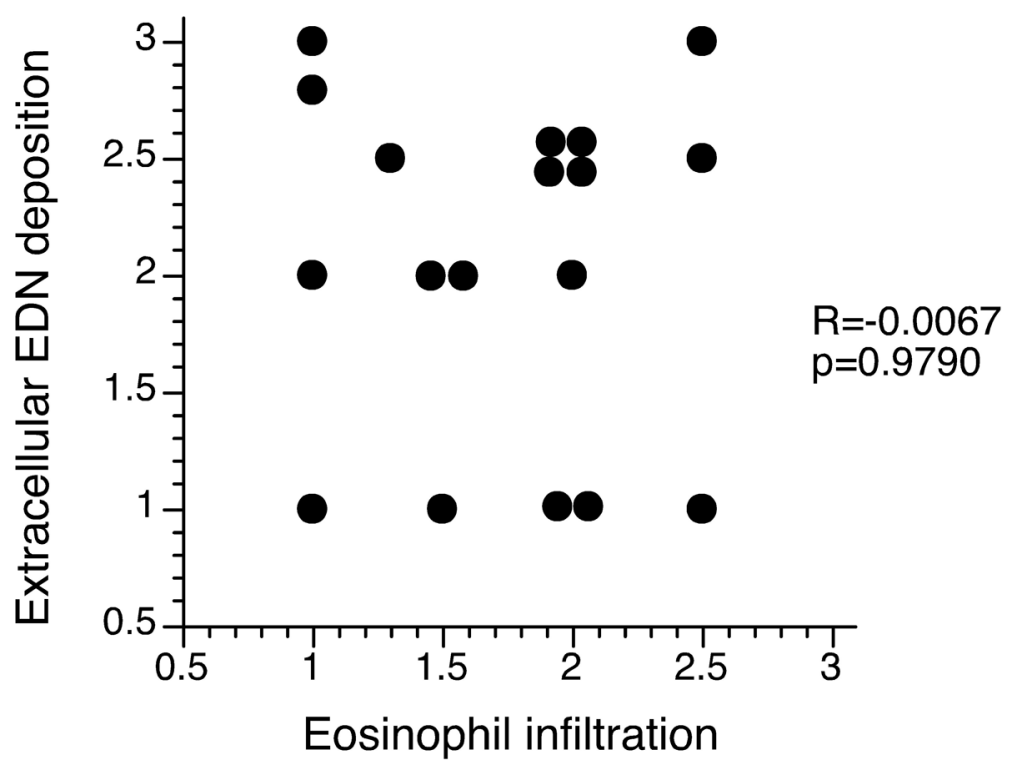

Figure 6.

Correlation between eosinophil infiltration scores and extracellular EDN deposition scores in EoE patients. Each dot represents a mean score from two independent observers for each biopsy. 


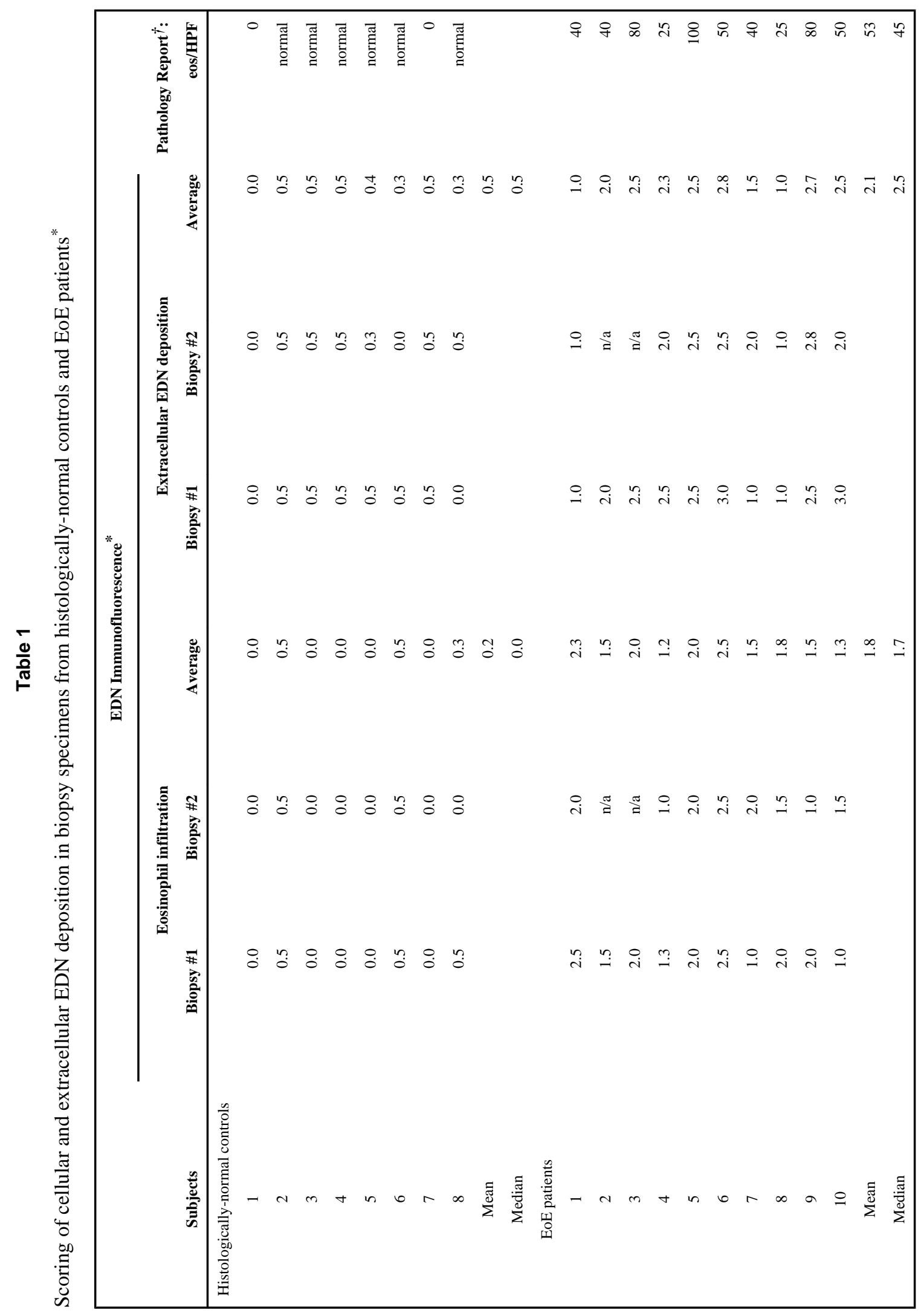

Am J Gastroenterol. Author manuscript; available in PMC 2010 August 1. 


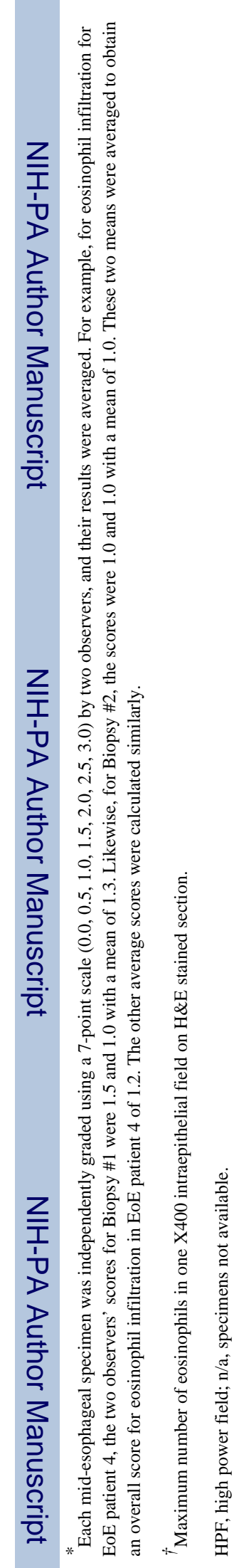

Am J Gastroenterol. Author manuscript; available in PMC 2010 August 1. 